\title{
Faringocoloplastia como reconstrução tardia pós-faringolaringectomia total por carcinoma espinocelular de hipofaringe: relato de caso e revisão da literatura
}

\author{
Pharyngocoloplasty as late reconstruction post-pharyngolaryngectomy by hypopharynx
} squamous cell carcinoma: case report and literature review

Leandro Luongo de Matos', Jossi Ledo Kanda', Fabio Roberto Pinto', Jaques Waisberg², Alexandre Cruz Henriques²

\begin{abstract}
Resumo
Introdução: 0 carcinoma espinocelular de cabeça e pescoço representa cerca de $5 \%$ de todas as neoplasias malignas. A localização dessa neoplasia no trato aerodigestivo superior é um fator prognóstico importante, sendo que, na maioria dos casos de hipofaringe, apresentam-se já avançados no momento do diagnóstico. A ressecção cirúrgica, quimioterapia e radioterapia adjuvantes têm grande importância no seu tratamento. Muitos pacientes, com controle loco-regional da doença, beneficiam-se de procedimentos cirúrgicos reparadores e de reconstrução do trânsito intestinal. Relato de caso: Homem, 38 anos, ex-etilista e ex-tabagista, com queixa de disfagia e dispneia rapidamente progressivas, submetido à traqueostomia de urgência por insuficiência respiratória aguda. À investigação, foi diagnosticado um carcinoma espinocelular de supraglote, com extensão para a parede medial do recesso piriforme esquerdo. 0 paciente foi, então, submetido à faringolaringectomia total, seguida de quimiorradiação. Sem sinais de recidiva tumoral após 12 meses de acompanhamento, foi realizada faringocoloplastia como reconstrução do trânsito intestinal. Encontrase atualmente em acompanhamento ambulatorial sem evidência de recidiva tumoral e satisfeito com o resultado cirúrgico final. Discussão: A reconstrução do trânsito intestinal em pacientes submetidos à faringolaringectomias é indicada para pacientes com controle local da doença e que aceitem o procedimento cirúrgico. A técnica mais utilizada é a interposição de alça de delgado. Porém, o cólon também é utilizado como substituto do esôfago. A escolha da técnica depende fundamentalmente da acurácia técnica do cirurgião e da necessidade de uma longa alça para anastomose entre a faringe e o estômago, com resultados satisfatórios.
\end{abstract}

Palavras-chave: Esofagocoloplastia; faringolaringectomia; tumores de cabeça e pescoço; carcinoma espinocelular.

\begin{abstract}
Introduction: Head and neck squamous cell carcinoma represents about $5 \%$ of all malignant tumors. Localization of this neoplasia at the upper aerodigestive tract is an important prognostic factor. In most cases, hypopharynx tumors are at an advanced stage when diagnosed. Surgical resection, as well as alongside chemotherapy and radiotherapy, are essential to the treatment. Many patients with locoregional control of the disease benefit from reparative and reconstructive surgeries of the intestinal transit. Case report: Man, 38 years old, former user of alcohol and tobaco, complaining of rapid progressive dysphagia and dyspnea, submitted to urgent tracheotomy due to acute respiratory failure. At examination, we diagnosed supraglottic squamous cell carcinoma, involving the left pyriform fossa medial wall. The patient underwent a pharyngolaryngectomy, followed by chemoradiation. Without signs of tumor recurrence after a 12-month follow-up, we performed a pharyngocoloplasty with reconstruction of the intestinal transit. At the moment, he is attending for an outpatient follow-up, with no evidences of tumor recurrence, and satisfied with the final surgical outcome. Discussion: The reconstruction of intestinal transit in pharyngolaryngectomy patients is indicated for those with local control of the disease and that accept this surgical procedure. The most used technique is the loop interposition of small intestine, but the colon is also used for esophageal replacement. Its choice depends mainly on the technical accuracy of the surgeon, but also on the requirement of a lengthy loop for the anastomosis between pharynx and the stomach, with suitable results.
\end{abstract}

Keywords: Esophagocoloplasty; pharyngolaryngectomy; head and neck tumors; squamous cell carcinoma.

Recebido: $18 / 09 / 2008$

Revisado: 11/05/2009

Aprovado: 11/05/2009

\footnotetext{
Estudo realizado no Hospital de Ensino Padre Anchieta, São Bernardo do Campo (SP), Brasil

Disciplina de Cirurgia de Cabeça e Pescoço da Faculdade de Medicina do ABC (FMABC), Santo André (SP), Brasil

Disciplina de Cirurgia do Aparelho Digestivo da Faculdade de Medicina do ABC (FMABC), Santo André (SP), Brasil

Endereço para correspondência: Alexandre Cruz Henriques - Rua Mediterrâneo, 928 - Jardim do Mar - CEP 09750-420 - São Bernardo do Campo (SP)

- Brasil - Tel: (11) 4121-9861 -E-mail: achenriques@uol.com.br
} 


\section{Introdução}

Os tumores de cabeça e pescoço representam menos de $5 \%$ de todas as neoplasias malignas e aproximadamente 3\% de todas as mortes por câncer nos Estados Unidos ${ }^{1}$. Mais de $90 \%$ dos casos, são histologicamente classificados como carcinomas espinocelulares (CEC) ${ }^{2}$. A localização dessa neoplasia no trato aerodigestivo superior é um fator prognóstico importante. Cerca de $80 \%$ dos tumores de hipofaringe, apresentam-se já avançados no momento do diagnóstico (estádio III e IV) ${ }^{3}$. O seu tratamento é complexo e multidisciplinar, tendo grande importância a ressecção cirúrgica, que em muitos casos pode ser extensa, e incluir, além do tumor primário, o esvaziamento linfonodal. Às vezes, a ressecção cirúrgica pode incluir também complexas técnicas de restabelecimento do trânsito alimentar, tais como: faringogastroplastia, faringocoloplastia ou transplante de jejuno com anastomose microcirúrgica.

Esse trabalho tem o objetivo de relatar um caso de doente portador de CEC avançado da hipofaringe que necessitou de ressecção alargada incluindo o esôfago cervical, posteriormente submetido à faringocoloplastia para restabelecimento do trânsito gastrointestinal.

\section{Relato do caso}

Homem, 38 anos, pardo, pedreiro, com queixa de rouquidão progressiva há quatro meses, acompanhada de disfagia e dispneia rapida-

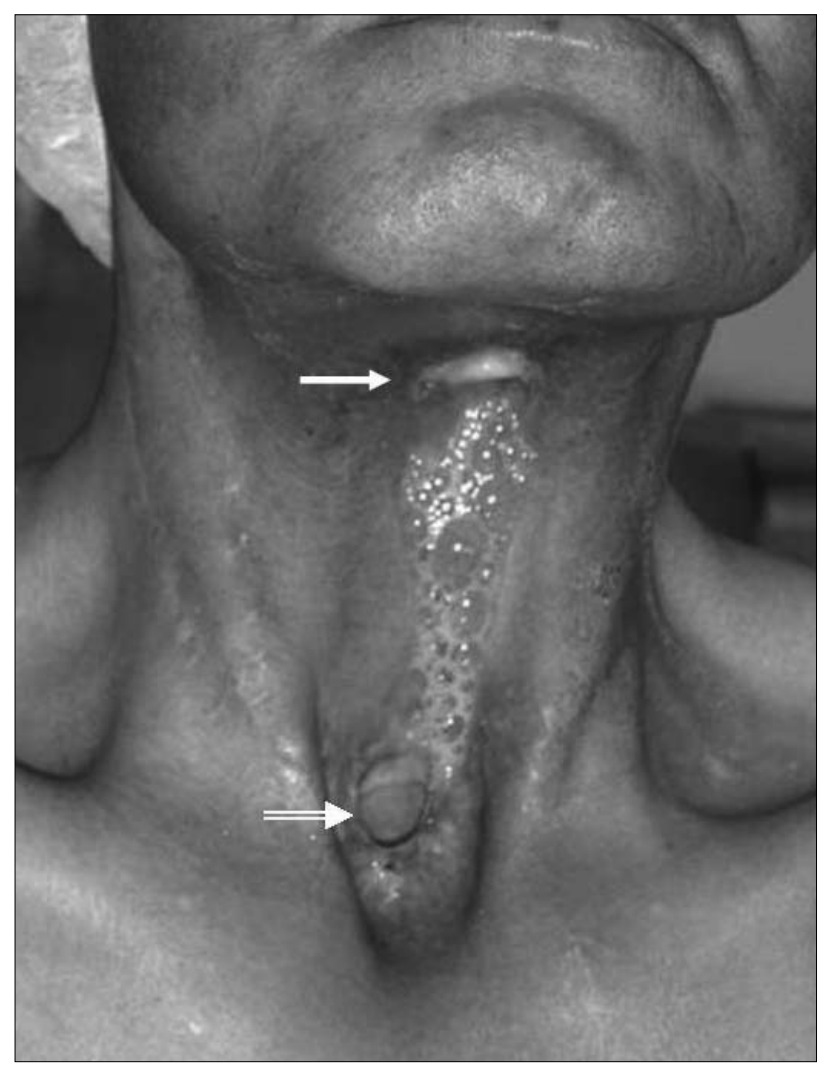

Figura 1 - Quinto mês pós-operatório de faringolaringectomia total por carcinoma espinocelular de hipofaringe. Seta simples: faringostomia; seta dupla: traqueostoma. mente progressivas. Há 12 dias, apresentou episódio de insuficiência respiratória aguda, necessitando de traqueostomia de urgência. Refere ser ex-tabagista e ex-etilista.

À fibrolaringoscopia foi identificada uma lesão úlcero-vegetante de supraglote, impedindo a visibilização das pregas vocais, com extensão para o recesso piriforme esquerdo. $\mathrm{O}$ exame anátomo-patológico de biópsia revelou um carcinoma espinocelular. O estadiamento préoperatório não evidenciou sinais clínicos ou radiológicos de doença disseminada, sendo indicado, portanto, o tratamento cirúrgico.

No intraoperatório foi encontrado tumor extenso de supraglote comprometendo o recesso piriforme esquerdo da hipofaringe e o esôfago proximal, sendo realizada a faringolaringectomia total; esofagectomia cervical; sepultamento intratorácico do esôfago distal; faringostomia; traqueostomia e esvaziamento cervical radical modificado à esquerda, e júgulo-carotídeo à direita, além de gastrostomia para alimentação.

O exame anátomo-patológico da peça cirúrgica revelou tratar-se de um tumor úlcero-infiltrativo, transglótico, de 50 × $35 \mathrm{~mm}$ nos maiores eixos, com destruição de ambas as pregas vocais, extensão para recesso piriforme esquerdo e esôfago proximal. A microscopia confirmou o diagnóstico de carcinoma espinocelular moderadamente diferenciado com infiltração perineural e sem comprometimento angiolinfático evidente. As margens cirúrgicas estavam livres de comprometimento neoplásico. Foram isolados 42 linfonodos do esvaziamento cervical esquerdo e 26 do direito, sendo identificado um linfonodo cervical metastático no grupamento jugulo-digástrico superior esquerdo (nível II). O estadiamento anátomo-patológico, portanto, foi T4N1Mx. Assim, o paciente foi submetido à radioterapia locoregional, associada à quimioterapia adjuvante.

Em 12 meses de evolução pós-operatória (Figura 1), o paciente apresentava-se clinicamente em bom estado e sem sinais de recidiva loco-regional ou de metástases hematogênicas, sendo indicada a reconstrução do trânsito alimentar. Foi submetido à faringocoloplastia com utilização do cólon transverso, mantendo-se a artéria cólica esquerda como pedículo vascular (Figura 2), rematuração da traqueostomia e rotação de retalho delto-peitoral esquerdo (Bakamjian) para recobrir o cólon transposto, uma vez que a pele local era inelástica, por ter sido previamente irradiada (Figura 3).

O paciente apresentou boa evolução pós-operatória, e recebeu alta no décimo segundo dia de pós-operatório. Após 48 meses da primeira operação, apresentava-se satisfeito com o resultado cirúrgico final. Permanece sem evidências de recidiva (Figura 4). Em virtude do risco de segundo tumor primário no esôfago remanescente, inacessível de acompanhamento endoscópico, foi proposta a esofagectomia, porém, o procedimento cirúrgico foi recusado pelo paciente.

\section{Discussão}

Em todo o mundo, estima-se que aproximadamente 644.000 novos casos de tumores de cabeça e pescoço sejam diagnosticados a cada ano ${ }^{1}$. Sua incidência é três vezes superior em homens que em mulheres 
e também é mais comum em afro-descendentes que em caucasianos. $\mathrm{O}$ carcinoma espinocelular é o tipo histológico mais prevalente e tabagismo e etilismo são os fatores etiológicos mais comuns ${ }^{4,5}$.

A apresentação clínica dos tumores de cabeça e pescoço depende de sua localização e extensão ${ }^{6}$. Como no caso descrito, as neoplasias de hipofaringe frequentemente cursam com disfagia, otalgia e rouquidão.

Lesões suspeitas nas vias aereodigestivas superiores ou linfonodos cervicais com características malignas devem ser investigadas com panendoscopia (nasofaringolaringoscopia, esofagoscopia e broncoscopia) e biópsias7. Quando o diagnóstico é estabelecido, a extensão local e regional da doença deve ser determinada, com intenção de adequado estadiamento. Exames de imagem podem ser utilizados: tomografia computadorizada de alta resolução para avaliação cervical e o estudo do tórax para detecção de metástases pulmonares ou eventual segundo tumor primário 8 .

O tratamento do câncer de cabeça e pescoço requer uma equipe multidisciplinar experiente, composta de cirurgião de cabeça e pescoço, radioterapeuta, oncologista, fonoaudiólogo, fisioterapeuta, e algumas vezes, do cirurgião do aparelho digestivo.

Os tumores precoces ( $\mathrm{T} 1$ ou $\mathrm{T} 2$, sem comprometimento linfonodal) são melhores tratados com cirurgias conservadoras com preservação do órgão ou radioterapia, dependendo da experiência da equipe. Os cânceres de estádio intermediário (tumores infiltrativos, T2 de pior prognóstico, T3 exofítico, N0-N1) beneficiam-se de tratamento combinado. Nos casos dos tumores avançados da hipofaringe, a faringolaringectomia total, seguida de radioterapia e quimioterapia adjuvantes, é opção terapêutica ${ }^{3,10-12}$. Algumas vezes, a reconstrução do trânsito alimentar e a correção do defeito estético tornam-se problemas desafiadores.

A reconstrução do trânsito alimentar é indicada para pacientes com controle local da doença e que aceitem o procedimento cirúrgico, que não é isento de complicações. Uma técnica muito empregada mundialmente é a interposição de retalhos livres entéricos ou cutâneos
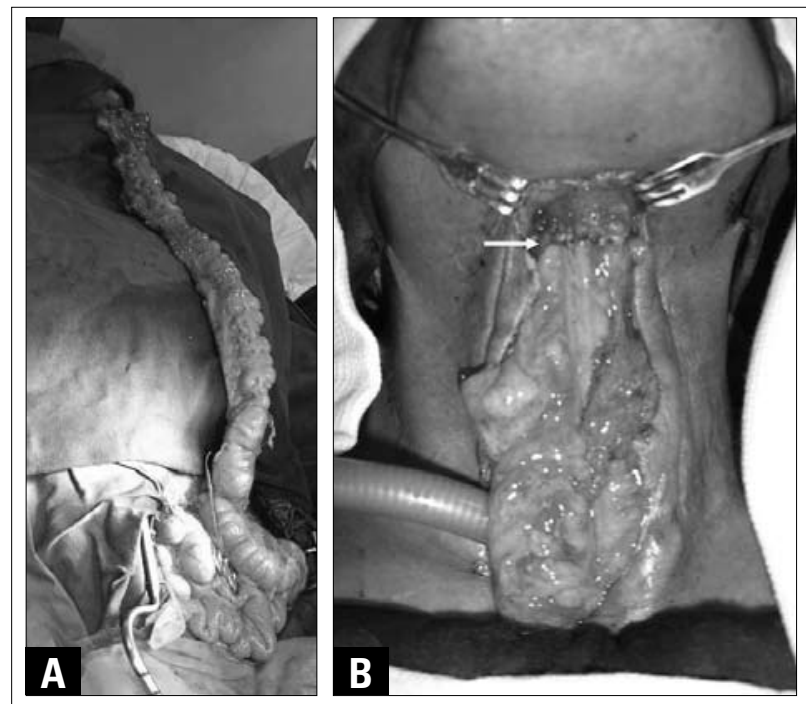

Figura 2 - Faringocoloplastia: (A) liberação do cólon transverso; (B) faringocolo-anastomose cervical anterior. Seta: anastomose faringo-cólica. em tubo ${ }^{13,14}$, que foram descartados, no presente caso, uma vez que o segmento faringoesofágico ressecado era muito longo.

O estômago e o cólon têm sido usados como substituto do esôfago desde $1911^{15}$. O estômago é o conduto de escolha pela facilidade na mobilização e o amplo aporte vascular ${ }^{16}$, porém, a reconstrução com transposição gástrica foi inviabilizada, no caso apresentado, em virtude da gastrostomia realizada previamente. O cólon (Figura 5) é utilizado quando o paciente foi previamente submetido à gastrostomia, gastrectomia parcial ou total e também quando há necessidade de uma longa alça para anastomose entre a faringe e o estômago ${ }^{16,17}$. Notase incidência mais elevada na mortalidade com o uso do cólon, uma vez que requer três anastomoses ${ }^{17}$. A perfusão do cólon ocorre por meio de uma vasta arcada vascular. Dessa forma, vários segmentos do cólon podem ser utilizados como substitutos do esôfago, como por exemplo, o cólon direito perfundido pela artéria cólica média e os cólons esquerdo ou transverso, como no caso relatado, perfundidos pela
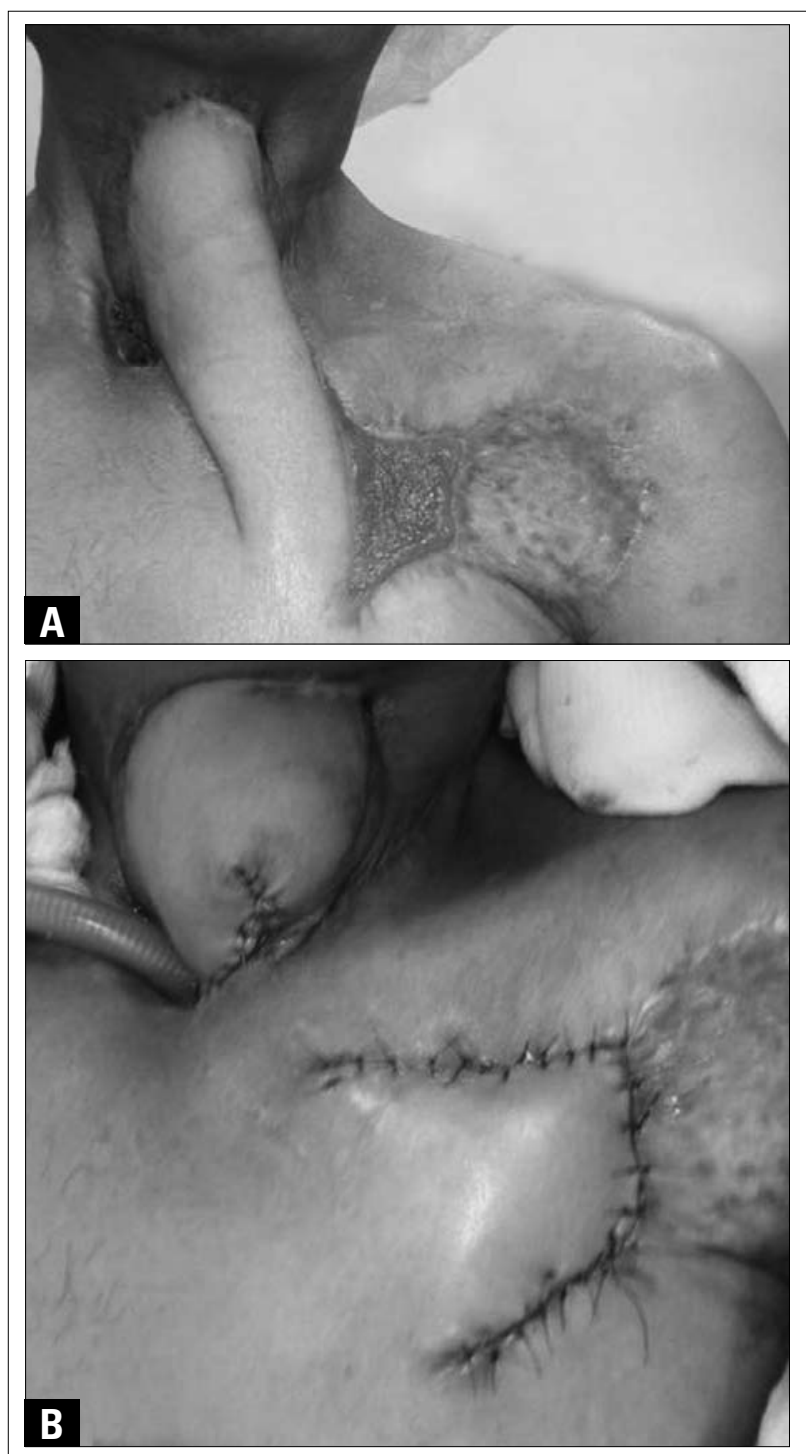

Figura 3 - (A) Retalho delto-peitoral esquerdo (a Bakamjian); (B) secção do mesmo em um segundo tempo (cerca de 45 dias). 


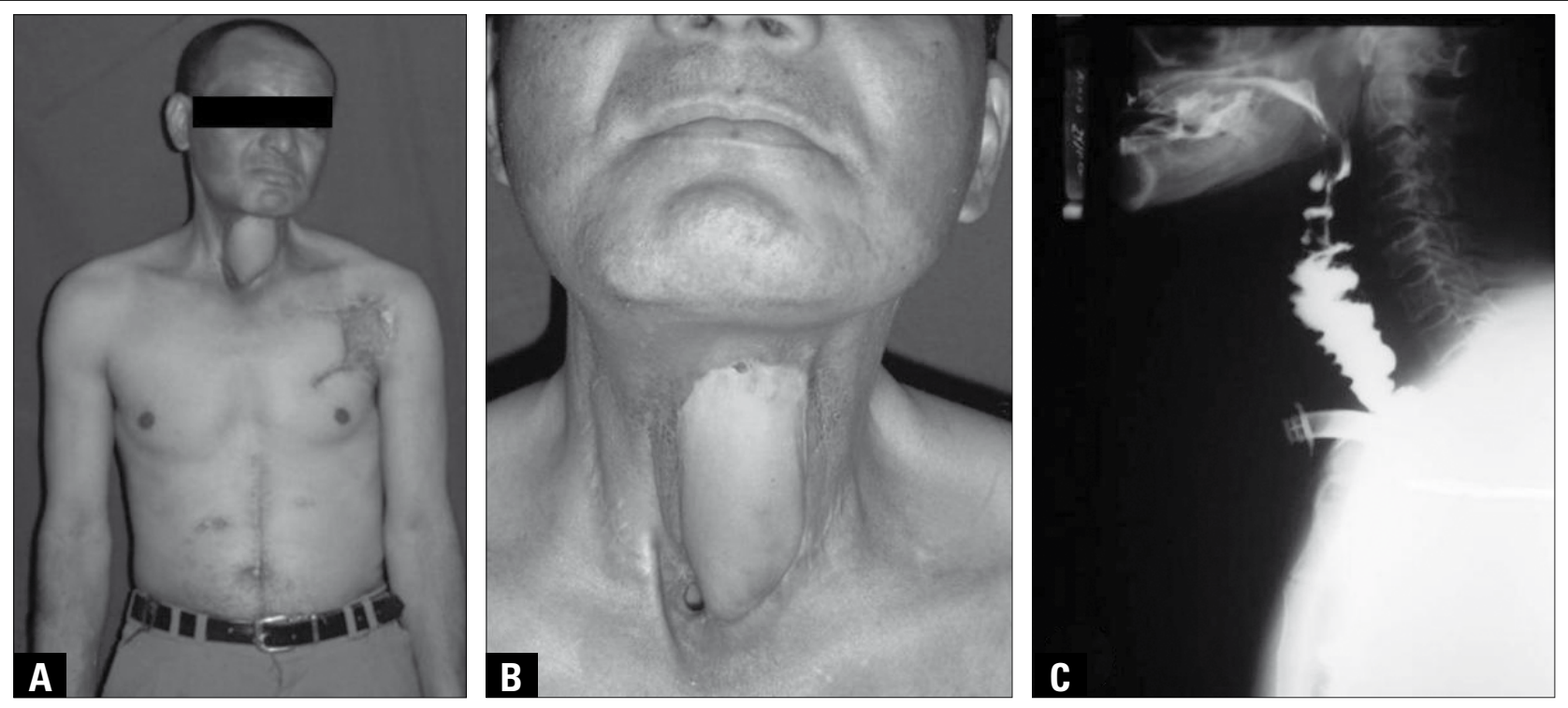

Figura 4 - Pós-operatório tardio. (A e B) Aspecto cervical definitivo; (C) deglutograma cervical sem evidência de fístula.

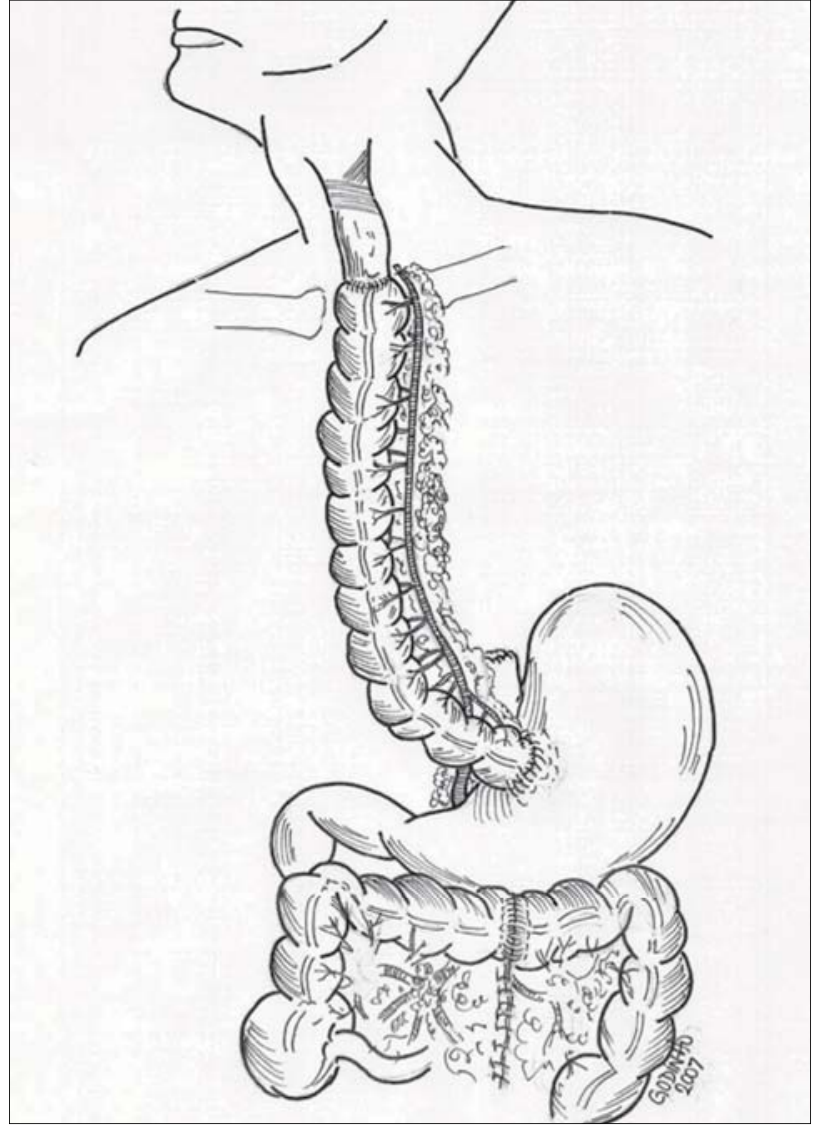

Figura 5 - Esquema representativo da esofagocoloplastia. Ilustração gentilmente cedida pelo Dr. Carlos Alberto Godinho

artéria cólica esquerda. O cólon também pode ser utilizado para substituir o esôfago em doenças benignas, como a estenose cáustica ${ }^{18}$.

As cirurgias tardias em pacientes faringolaringectomizados para reconstrução do trânsito alimentar, frequentemente ocasionam defeitos cutâneos. Uma opção muito utilizada como cobertura cutânea é o re- talho delto-peitoral (descrito por Bakamjian), com resultados estéticos satisfatórios ${ }^{19}$. Este foi indicado no caso descrito, devido a fibrose local pós-radioterapia que impedia o deslizamento de retalhos locais.

Pacientes com tumores avançados de hipofaringe podem apresentar tumores sincrônicos ou metacrônicos. Estudo sobre a incidência de segundo tumor primário em esôfago em 152 pacientes com carcinomas espinocelulares de mucosa da região de cabeça e pescoço identificou oito $(5,3 \%)$ neoplasias sincrônicas e seis $(2,6 \%)$ metacrônicas durante seguimento de até sete anos ${ }^{16}$. No caso apresentado, o remanescente esofágico, por estar excluso do trânsito alimentar, não é acessível ao estudo endoscópico. Nesses casos, a esofagectomia total profilática pode ser indicada em um segundo tempo ${ }^{17}$. O paciente desse estudo recusou-se a ser submetido a mais um procedimento cirúrgico. Está sendo acompanhado ambulatorialmente, e realiza tomografia computadorizada de mediastino periodicamente para deteç̧ão de eventual segundo tumor primário.

A taxa de sobrevivência nos pacientes com carcinomas espinocelulares de cabeça e pescoço, em cinco anos, varia de 40 a 64\%, dependendo da sua localização e principalmente do estadiamento ${ }^{4}$. Um estudo retrospectivo recente aponta especificamente para pacientes com tumores de hipofaringe submetidos à faringolaringectomia total $\mathrm{e}$ terapia adjuvante (tumores de estádio I e II), sobrevivência global acumulada em dois e cinco anos, de 68 e $53 \%$, respectivamente ${ }^{12}$. Os casos de tumores avançados de hipofaringe (T3 ou T4) apresentam 29\% de sobrevivência em cinco anos e o comprometimento do esôfago cervical diminui essa taxa para $14 \%{ }^{20}$.

Em casos selecionados julgamos indicada a realização de grandes ressecções, mesmo que sejam necessárias complexas técnicas de reconstituição do trânsito alimentar e de confecção de retalhos para diminuir o defeito estético. Tal abordagem representa uma opção para controle loco-regional da doença com melhora na qualidade de vida para esses pacientes. 


\section{Referências}

1. Jemal A, Siegel R, Ward E, Hao Y, Xu J, Murray T, et al. Cancer statistics, 2008. CA Cancer J Clin 2008;58(2):71-96.

2. Stabenow E, Moysés RA, Paraventi C, Montenegro FLM, Tavares MR, Araújo Filho VJF, et al. Metástase linfática cervical no carcinoma espinocelular de vias aerodigestivas superiores: análise crítica de fatores preditivos, pelo modelo de regressão logística. Rev Bras Cir Cabeça Pescoço 2007:36(4):202-8

3. Iseli TA, Agar NJ, Dunemann C, Lyons BM. Functional outcomes following total laryngopharyngectomy. ANZ J Surg 2007;77(11):954-7.

4. Hayat MJ, Howlader N, Reichman ME, Edwards BK. Cancer statistics, trends, and multiple primary cancer analyses from the Surveillance, Epidemiology, and End Results (SEER) Program. Oncologist 2007;12(1):20-37.

5. Goldenberg D, Lee J, Koch WM, Kim MM, Trink B, Sidransky D, et al. Habitual risk factors for head and neck cancer. Otolaryngol Head Neck Surg 2004;131(6):986-93.

6. Suen JY, Stern SJ. Cancer of the Head and Neck. In: Myers EN, Suen JY, editors. Cancer of the Head and Neck. 1st ed. Philadelphia: WB Saunders: 1996. p. $462-84$

7. Stoeckli SJ, Zimmermann R, Schmid S. Role of routine panendoscopy in cancer of the upper aerodigestive tract. Otolaryngol Head Neck Surg 2001;124(2):208-12.

8. van den Brekel MW, Castelijns JA, Snow GB. The role of modern imaging studies in staging and therapy of head and neck neoplasms. Semin Oncol 1994;21(3):340-8

9. Adams S, Baum RP, Stuckensen T, Bitter K, Hor G. Prospective comparison of 18F-FDG PET with conventional imaging modalities (CT, MRI, US) in lymph node staging of head and neck cancer. Eur J Nucl Med 1998;25(9):1255-60.

10. Posner M. Options in the Treatment of Head and Neck Cancer. The Oncologist Group. Manhasset: CMPMedica: 2006. p. 1-122.
11. Panizza B. Pharyngolaryngectomy: still a necessary surgical evil. ANZ J Surg 2007:77(11):932.

12. Bova R, Goh R, Poulson M, Coman WB. Total pharyngolaryngectomy for squamous cell carcinoma of the hypopharynx: a review. Laryngoscope 2005;115(5):864-9.

13. Pesko P, Sabliak P, Bjelovic M, Stojakov D, Simic A, Nenadic B, et al. Surgical treatment and clinical course of patients with hypopharyngeal carcinoma. Dis Esophagus 2006;19(4):248-53.

14. Chen YB, Chen HC, Lee YC. Bakamjian island flap for patch esophagoplasty of the cervical esophagus. Plast Reconstr Surg 1999;103(4):1176-80.

15. Kelling G. Oesophagoplastik mit Hilfe des Querkolon. Zentrabl Chir 1911;38:1209-12

16. Ferreira EAB, Altmann DA0. Cirurgia do esôfago. In: Goffi FS, editor. Técnica cirúrgica: bases anatômicas, fisiopatológicas e técnicas da cirurgia. $1^{\mathrm{a}} \mathrm{ed}$. São Paulo: Atheneu: 2007. cap 68. p. 822.

17. Bussi M, Ferrero V, Riontino E, Gasparri G, Camandona M, Cortesina G. Problems in reconstructive surgery in the treatment of carcinoma of the hypopharyngoesophageal junction. J Surg Oncol 2000;74(2): 130-3

18. Rice TW. Colon replacement. In: Pearson FG, Cooper JD, Deslauriers J, Ginsberg RJ, Hiebert CA, Patterson GA, editors. Esophageal surgery. 2nd ed. Filadélfia: Churchill Livingstone: 2002. cap 65. p. 917-30.

19. Andrews BT, McCulloch TM, Funk GF, Graham SM, Hoffman HT. Deltopectoral flap revisited in the microvascular era: a singleinstitution 10-year experience. Ann Otol Rhinol Laryngol 2006;115(1): $35-40$

20. Triboulet JP, Mariette C, Chevalier D, Amrouni H. Surgical management of carcinoma of the hypopharynx and cervical esophagus: analysis of 209 cases. Arch Surg 2001;136(10):1164-70. 